\title{
FRACTIONAL BLOCH'S EQUATIONS APPROACH TO MAGNETIC RELAXATION*
}

\author{
ZENON MATUSZAK \\ Department of Medical Physics and Biophysics, AGH University of Science and Technology, Krakow,Poland
}

Received December 11, 2013; accepted February 26, 2014; published online March 07, 2014

\begin{abstract}
It is the goal of this paper to present general strategy for using fractional operators to model the magnetic relaxation in complex environments revealing time and spacial disorder. Such systems have anomalous temporal and spacial response (non-local interactions and long memory) compared to systems without disorder. The systems having no memory can be modeled by linear differential equations with constant coefficients (exponential relaxation); the differential equations governing the systems with memory are known as Fractional Order Differential Equations (FODE).

The relaxation of the spin system is best described phenomenologically by so-called Bloch's equations, which detail the rate of change of the magnetization M of the spin system. The Ordinary Order Bloch's Equations (OOBE) are a set of macroscopic differential equations of the first order describing the magnetization behavior under influence of static, varying magnetic fields and relaxation. It is assumed that spins relax along the $z$ axis and in the $x-y$ plane at different rates, designated as $R_{1}$ and $R_{2}$ $\left(R_{1}=1 / T_{1}, R_{2}=1 / T_{2}\right)$ respectively, but following first order kinetics.

To consider heterogeneity, complex structure, and memory effects in the relaxation process the Ordinary Order Bloch's Equations were generalized to Fractional Order Bloch's Equations (FOBE) through extension of the time derivative to fractional (non-integer) order.

To investigate systematically the influence of "fractionality" (power order of derivative) on the dynamics of the spin system a general approach was proposed. The OOBE and FOBE were successively solved using analytical (Laplace transform), semi-analytical (ADM - Adomian Decomposition Method) and numerical methods (Grünwald- Letnikov method for FOBE). Solutions of both OOBE and FOBE systems of equations were obtained for various sets of experimental parameters used in spin $1 / 2$ NMR and EPR spectroscopies. The physical meaning of the fractional relaxation in magnetic resonance is shortly discussed.
\end{abstract}

\section{INTRODUCTION}

A growing interest in investigations of structures and dynamics of complex systems has been observed during two past decades. The term complex systems covers such different systems as glasses, amorphous systems, colloids, microemulsions, polymers and biopolymers, organisms and societies etc. The three common features are characteristic for such systems: (a) a large diversity of elementary units, (b) strong interactions between the units, or (c) anomalous evolution in the course of time. Complex systems are disordered on a microscopic scale and homogeneous on macroscopic scale but often they possess the certain degree of order on intermediate, so called mesoscopic scale. It is due to specific balance between interactions and thermal motions. Such systems have anomalous temporal and spacial response (nonlocal interactions and long memory effects) compared to systems without disorder. It is known since XIX century that the simple exponential relaxation law cannot adequately describe the relaxation processes and kinetics in such systems; relaxation has often non-exponential (power law) character (Feldman, Puzenko \& Ryabov, 2006; Metzler, Klafter, 2000).

Nowadays, complex systems, are studied on all structural levels from microscopic to macroscopic using different experimental techniques, which are able to examine these systems at different levels of resolution, both, in a space and time. The area of investigation covers practically all fields of physics, engineering, chemistry, biophysics (West, 2006), and econophysics to mention some of them. Various aspect of physics of the complex phenomena are investigated based on fractional calculus approach. Fractional calculus being a kind of calculus, is a generalization of ordinary calculus in which the derivatives and integrals of arbitrary order are considered. There are systematically published special monographs discussing various applications of fractional calculus in solving physical problems (Herrmann, 2011; Uchaikin, 2013).

Experimental methods used to study complex systems should be sensitive to intermolecular interactions and

\footnotetext{
* This paper was presented at IX International Workshop on EPR (ESR) in Biology and Medicine, 7-10 October, 2013, Kraków, Poland
} 
cooperative interactions. The magnetic resonance techniques, both NMR and EPR (and combinatory techniques) satisfy these requirements, but up to now the potential of these methods was not exploited in systematic investigations of complex systems with memory and non-local interaction. Probably due to lack of unified theoretical description of magnetic resonance phenomena in such systems, although e.g. some experimental investigations of relaxation in fractal-like structures were undertaken in frames of ESR spectroscopy, as early as in 80-ties of XX century (Alexander, Entin - Wohlman \& Orbach, 1985; Alexander, Entin - Wohlman \&Orbach, 1986).

This situation had changed in last few years, when first papers describing applications of fractional calculus for solving fractional Bloch equations appeared in the scientific literature (Magin, Feng \& Baleanu, 2009). Fractional calculus seems to be exactly the natural mathematical language which describes the systems with memory and non-locality in an uniform way. The fractional calculus being a very old and simultaneously very young area of mathematical and scientific applications, probably would stay the calculus of XXI century, in many opinions, with systematically growing fields of applications (e.g. Sabatier, Agrawal, Tenreiro \& Machado, 2007).

It is the goal of this paper to present some aspects of a general strategy in solving Fractional Order Bloch's Equations (FOBE). In this presentation FOBE represent the description of magnetic relaxation in complex environments revealing time and spacial disorder on the phenomenological level. The presented approach concerns both analytical and simulation results.

\section{FRACTIONAL CALCULUS}

The concept of fractional calculus - that is - calculus of integrals and derivatives of arbitrary order has very old history in mathematics, can be traced back to 1695 (Oldham \& Spanier, 1974). It was developed simultaneously with the theory of classical calculus. But unlike classical derivatives and integrals, which have unique definitions and proper geometrical and physical interpretations for a long time, there are many definitions of fractional integrals and derivatives, while a single interpretation of fractional operators is lacking. A dozen or so definitions of fractional operators are known and used in the literature at present, three of which will be presented in this work. Because of the challenges on the theoretical level, practical applications of fractional calculus is a relatively new topic of research. The ideas of fractional calculus found many important applications in science, engineering and finance in the last three decades alone. Now the fractional calculus, involving fractional differential equations theory, is a rapidly growing area of pure and applied sciences. Some necessary terms and fundamental definitions concerning fractional calculus will be given further, omitting rigorous mathematics.

\section{Fractional Calculus-Basic Definitions}

Fractional calculus is a generalization of conventional calculus to non-integer order integral or derivative, and the mathematicians have found many ways to define the intuitive concept of the non-integer order derivative and integral (Oldham et al, 1974; Miller \& Ross, 1993).

Many of definitions used in fractional calculus are variations of so called Riemann-Liouville (RL) definition and this definition will be given as first below. The R-L approach is based on the generalization of classical Cauchy formula for repeated integration - the calculation of the n-fold integral is reduced to convolution integral. If we introduce the succinct notation the Cauchy formula can be written as follows:

$$
\begin{aligned}
& I^{n} f(t):=D^{(-n)} f(t)=\frac{1}{(n-1) !} \int_{a}^{t}(t-\tau)^{(n-1)} f(\tau) d \tau, \\
& t>0, n \in \square,
\end{aligned}
$$

where operator $I^{n}=D^{(-n)}$ denotes the integration of function $\mathrm{f}(\mathrm{t})$ to an order $\mathrm{n}$. The initial value a is usually set to be 0 .

Using the known properties of Euler's Gamma function $\Gamma(\mathrm{z})$ :

$$
\begin{aligned}
& \Gamma(z)=\int_{0}^{\infty} t^{z-1} e^{-t} d t, \\
& \Gamma(z+1)=z \Gamma(z),
\end{aligned}
$$

$n !=\Gamma(n+1)$,

where the integral converges for $\operatorname{Re}(\mathrm{z})>0$, the Cauchy's formula may be extend to any positive real values and defines the fractional integral of Riemann-Liouville type order $\alpha>0$ :

$$
I^{\alpha} f(t):=D^{(-\alpha)} f(t)=\frac{1}{\Gamma(\alpha)} \int_{a}^{t}(t-\tau)^{(\alpha-1)} f(\tau) d \tau, t>0
$$

The fractional RL derivative of order $\alpha$ denoted here as ${ }^{\mathrm{RL}} \mathrm{D}^{\alpha}$ can be defined using the previously defined $\mathrm{RL}$ integral. If we introduce a positive integer $n$ chosen in such a way that $\mathrm{n}-1<\alpha<\mathrm{n}$ then ${ }^{\mathrm{RL}} \mathrm{D}^{\alpha}$ is defined as:

$$
{ }_{a}^{R L} D_{t}^{\alpha} f(t)=\frac{1}{\Gamma(n-\alpha)} \frac{d^{n}}{d t^{n}} \int_{a}^{t} \frac{f(\tau)}{(t-\tau)^{(\alpha-n+1)}} d \tau,
$$


and additionally an operational equation is coming true:

$$
{ }_{a}^{R L}{ }_{t}^{\alpha}\left(I^{\alpha} f\right)(x)=f(x)
$$

The next type of fractional derivative is so called Caputo fractional derivative ${ }^{\mathrm{C}} \mathrm{D}^{\alpha}$ defined as follows:

$$
{ }_{a}^{C} D_{t}^{\alpha} f(t)=\frac{1}{\Gamma(n-\alpha)} \int_{a}^{t} \frac{f^{(n)}(\tau)}{(t-\tau)^{(\alpha-n+1)}} d \tau
$$

where $\mathrm{f}^{(\mathrm{n})}$ denotes the $\mathrm{n}$-th derivative of function $\mathrm{f}$ and as previously $n-1<\alpha<\mathrm{n}$. In Caputo's derivative, the order of integration and differentiation is changed comparing to $\mathrm{R}-\mathrm{L}$ derivative. Caputo derivative is "fractional integration of a derivative of the function" but R-L derivative is " the derivative of fractional integral".

The Caputo derivatives are often used in applications because they have properties similar that we are familiar in conventional calculus.

It is seen from eqs. $(4,5)$ that the fractional derivatives have non-local character, in opposition to usual derivatives which are local operators. They involve the integration as a vital part of differentiation operation; that means that the calculation of a derivative demands the knowledge about values of the function in the whole interval. Therefore, to underline this property, fractional derivatives and integrals are often described by the general term differintegrals.

The definition of Grünwald-Letnikov ${ }^{\mathrm{GL}} \mathrm{D}^{\alpha}$ fractional derivative approaches from derivative side. In this approach fractional derivative of order $\alpha$ is the straightforward generalization of the definition for usual derivative of integer order. The final formula for ${ }^{\mathrm{GL}} \mathrm{D}^{\alpha}$ has the form:

$$
{ }_{a}^{G L} D_{t}^{\alpha} f(t)=\lim _{h \rightarrow 0} \frac{1}{h^{\alpha}} \sum_{j=0}^{\left[n=\frac{t-a}{h}\right]}(-1)^{j} \frac{\Gamma(\alpha+1)}{\Gamma(j+1) \Gamma(\alpha-j+1)} f(t-j h)
$$

where $\mathrm{t}$ and a are the lower and upper limits of differentiation, respectively; and [ ] denotes the integer part (floor function), which maps the real number to smallest following integer.

Rewriting (6) for $(-\alpha)$ we obtain the GrünwaldLetnikov (GL) integral:

$$
{ }_{a}^{G L} D_{t}^{-\alpha} f(t)=\lim _{h \rightarrow 0} h^{\alpha} \sum_{j=0}^{\left[n=\frac{t-a}{h}\right]}(-1)^{j} \frac{\Gamma(\alpha+j)}{\Gamma(j+1) \Gamma(\alpha)} f(t-j h) .
$$

The GL approach is mostly used in construction of numerical algorithms for Fractional Differential
Equations (FDE). For many practical purposes, for a great variety of functions the RL, C, and GL definitions are equivalent. The general introduction to fractional calculus can be found in cited monographs e.g.(Oldham et al., 1974, Miller at al., 1993; Uchaikin, 2013).

\section{Fractional Differential Equations}

Fractional Differential Equations (FDE) are the class of differential equations in which the usual derivatives of integer order (ordinary or partial) were replaced by fractional derivatives. Currently such equations have a very rich theory, the results and applications of FDE are discussed in many books (Podlubny, 1999; Kilbas, Srivastava \& Trujillo, 2006; Petráš, 2011).

Only Initial Value Problems (IVP) - Cauchy type problems on a finite interval for Fractional Ordinary Differential Equations (FODE) of real order will be considered here because the FOBE belong to this category of equations.

The problem of initial conditions is rather subtle in physical applications of FODE because the applications of FODE require well defined initial conditions having physical interpretation.

As mentioned above there exist many kinds of fractional derivatives. It is worthy to note at this place that the RL derivative of the constant function is not 0 (Podlubny, 1999). This fact results in difficulties in the physical interpretation of initial conditions for RL derivatives. Initial conditions must include the limit values of the RL derivatives at the lower terminal $t=a$; however, the physical interpretation of such type of conditions is not known (Podlubny, 1999).

This problem does not appear when we use Caputo's derivative. For FDE with Caputo derivative, the initial conditions take on the same form as for Integer-Order Differential Equations (IODE) (Diethelm, 2010). This fact also facilitates the use of operator calculus to solve FDE analytically. Best fitted to this purpose are Laplace and Fourier transforms, but other integral transforms are also effective (Podlubny, 1999; Monje, Chen., Vinagre, Xue \& Feliu, 2010; Kaczorek, 2011). The general problem of initial conditions (and boundary conditions) for FDE, known as a problem of initialization for FDE, was systematically studied from different points of views, some aspects are discussed in cited literature (Podlubny, 1999; Petráš, 2011; Butzer \& Westphal, 2000; Hilfer, 2000).

Analytical solutions of FODE are difficult to receive generally (Kilbas at al., 2006), in most cases the numerical methods must be used. Many numerical techniques for solving FDE were elaborated in recent times. Most frequently they are modifications of generally known discretization scheme previously developed for solving IODE, but some additional problems not known in IODE appear in solving FDE due to non-local character of fractional derivatives 
(Weilbeer, 2005; Diethelm, 2010). Generally, the approximation of fractional derivatives is non-trivial problem. The special numerical techniques were worked out to perform the approximation of fractional derivatives with desired accuracy, including so-called frequency domain approach, originally developed to solve fractional control theory problems and very useful in a number of practical applications (Oustaloup, Mathieu \& Lanusse, 1995; Duarte, 2005).

\section{BLOCH'S EQUATIONS}

The Bloch's equations give a simple qualitatively and quantitatively description of both $\mathrm{cw}$ and pulsed magnetic resonance experiments on $\mathrm{S}=1 / 2$ systems. The set of Bloch's equations (in a laboratory frame) describing the motion of magnetization $\mathbf{M}=$ $\left(\mathrm{M}_{\mathrm{x}}, \mathrm{M}_{\mathrm{y}}, \mathrm{M}_{\mathrm{z}}\right)^{\mathrm{T}}$ in static $\mathbf{B}_{0}$ magnetic field (Zeeman field) and radiofrequency (or microwave in the case of ESR) fields is given below (eqs. $8 \mathrm{a}, \mathrm{b}, \mathrm{c}$ ). $\mathbf{M}_{0}$ is the equilibrium $\mathbf{z}$ magnetization.

Due to interaction with static magnetic field $\mathbf{B}_{0}=\mathrm{B}_{0} \mathbf{z}$ the magnetization vector precesses around $\mathbf{B}_{0}$ field direction with Larmor frequency $\omega_{0}=\gamma\left|B_{0}\right|, \gamma$ is the gyromagnetic ratio and for proton $\gamma=42.52 \mathrm{MHz} /$ Tesla (for simplicity the NMR convention concerning $\mathrm{H}$ nuclei will be used throughout the text). After sample is placing in a magnetic field, the magnetization goes to thermal equilibrium with no transverse components. To detect the magnetic resonance signal the magnetization is perturbed away from thermal equilibrium by additional oscillating magnetic field $\mathbf{B}_{1}=\mathrm{B}_{1} \cos \left(\omega_{1} \mathrm{t}\right)$ perpendicular to $\mathbf{B}_{\mathbf{0}}$, creating oscillating field in the sample; $\omega_{1}$ is a frequency of radio(microwave) oscillating field. The general magnetic field has the form $\mathbf{B}=\mathbf{B}_{\mathbf{0}}+\mathbf{B}_{\mathbf{1}}(\mathrm{t})$. The $\mathbf{B}_{\mathbf{1}}(\mathrm{t})$ field can be decomposed into two counter- rotating fields: $\mathbf{B}_{1,2}(\mathrm{t})=\mathrm{B}_{1} \cos \left(\omega_{1} \mathrm{t}\right) \mathbf{x} \pm$ $B_{1} \sin \left(\omega_{1} t\right) \mathbf{y}$, one or the other of these fields will always be rotating in the same sense as the precession of spins and affects the spins. Relaxation processes are included in a purely phenomenological manner in this model, by introducing a longitudinal (spin-lattice) relaxation time $\mathrm{T}_{1}$ and a transverse (spin-spin) relaxation time $\mathrm{T}_{2}$, assuming exponential character of relaxation in both cases. The result are Bloch's equations (Haacke, Brown, Thompson \& Venkatesan, 1999; Slichter, 1989):

$$
\frac{d \vec{M}}{d t}=\gamma \vec{M} \times \vec{B}-\frac{\left(M_{x} \hat{i}_{x}+M_{y} \hat{i}_{y}\right)}{T_{2}}-\frac{\left(M_{z}-M_{0}\right)}{T_{1}} \hat{i}_{z} .
$$

For components of magnetization, we have:

$$
\frac{d M_{x}}{d t}=-\omega_{0} M_{y}+\gamma B_{1} \sin \left(\omega_{1} t\right) M_{z}-\frac{1}{T_{2}} M_{x},
$$

$$
\begin{aligned}
& \frac{d M_{y}}{d t}=\omega_{0} M_{x}+\gamma B_{1} \cos \left(\omega_{1} t\right) M_{z}-\frac{1}{T_{2}} M_{y}, \\
& \frac{d M z}{d t}=-\gamma B_{1} \sin \left(\omega_{1} t\right) M_{x}+\gamma B_{1} \cos \left(\omega_{1} t\right) M_{y}-\frac{1}{T_{1}}\left(M_{z}-M_{0}\right)
\end{aligned}
$$

The solving of Bloch's equations given in their general form is rather difficult (the coefficients contain the oscillating terms). Known solutions have very complicated algebraic form, unsuitable for practical applications, as evidenced in (Torrey,1949; Yariv, 1975; Madhu, Kumar, 1995). In this work, the simplified form of Bloch's equations will be considered and solved, but the proposed method has a general character and may be generalized to solve analytically and numerically the Bloch's (and similar) equations for various sets of initial conditions.

\section{METHODS AND RESULTS STATE REPRESENTATION APPROACH}

State (state vector) representation is a routine approach used in optimal control theory (Lewis \& Syrmos, 1995; Kaczorek, 2011) and it may be generalized to solve fractional problems. Generally we search solution of the control problem (monovariable system of commensurate order in considered case) given in following form:

$$
\begin{aligned}
& D_{t}^{\alpha} \vec{x}(t)=\frac{d^{\alpha} \vec{x}}{d t^{\alpha}}=\mathscr{A} \vec{x}(t)+\boldsymbol{B} \vec{u}(t), 0 \leq \alpha \leq 1 \\
& \vec{y}(t)=\mathbb{C} \vec{x}(t)+\boldsymbol{D} \vec{u}(t),
\end{aligned}
$$

where $\mathbf{x}(\mathrm{t}) \in \mathbb{R}^{\mathrm{n}}, \mathbf{u}(\mathrm{t}) \in \mathbb{R}^{\mathrm{m}}, \mathbf{y}(\mathrm{t}) \in \mathbb{R}^{\mathrm{p}}$, matrices $\mathbb{A} \in \mathbb{R}^{\mathrm{nxn}}$, $\mathbb{B} \in \mathbb{R}^{\mathrm{nxm}}, \mathbb{C} \in \mathbb{R}^{\mathrm{pxn}}, \mathbb{D} \in \mathbb{R}^{\mathrm{pxm}} . \mathrm{D}_{\mathrm{t}}^{\alpha}$ can be an integer order derivative (when $\alpha=1$ ) or RL, or Caputo operator. Vectors $\mathbf{x}(\mathrm{t}) \mathbf{u}(\mathrm{t}), \mathbf{y}(\mathrm{t})$ are called state vectors, input and output respectively. Matrix $\mathbb{A}$ is state transition matrix (Monje at al., 2010; Kaczorek, 2011). The general solution of system can be written as:

$$
\vec{x}(t)=\Phi_{0} \vec{x}_{0}+\int_{0}^{t} \Phi(t-\tau) \boldsymbol{B} \vec{u}(\tau) d \tau, \vec{x}(0)=\vec{x}_{0}
$$

For $\alpha=1, \Phi_{0}(\mathrm{t})=\Phi(\mathrm{t})$ :

$$
\Phi_{0}(t)=\Phi(t)=\sum_{k=0}^{\infty} \frac{\mathscr{A}^{k}}{\Gamma(k+1)}=e^{\mathcal{A} t}
$$

where $\exp (\mathbb{A})$ is a exponential of matrix defined as: 


$$
\operatorname{Exp}(\boldsymbol{A})=e^{\mathcal{A}}:=\sum_{k=0}^{\infty} \frac{\boldsymbol{A}^{k}}{k !} .
$$

For $\alpha \neq 1$ we get:

$$
\Phi_{0}(t)=E_{\alpha}\left(\not \boldsymbol{A} t^{\alpha}\right)=\sum_{k=0}^{\infty} \frac{\boldsymbol{A}^{k} t^{k \alpha}}{\Gamma(k \alpha+1)},
$$

and

$$
\Phi(t)=\sum_{k=0}^{\infty} \frac{\boldsymbol{A}^{k} t^{(k+1) \alpha-1}}{\Gamma[(k+1) \alpha]},
$$

where $\mathrm{E}_{\alpha}\left(A \mathrm{t}^{\alpha}\right)$ is a matrix Mittag-Leffler function.

Mittag-Leffler (M-L) functions, which are generalizations of exponentials, play a fundamental role in fractional calculus because they are eigenfunctions of fractional operators (Podlubny, 1999). Mittag-Leffler functions of complex variable $\mathrm{z}$ can be written in following form (series representation): one parameter M-L function $\mathrm{E}_{\alpha}(\mathrm{z})$ :

$$
E_{\alpha}(z)=\sum_{k=0}^{\infty} \frac{z^{k}}{\Gamma(k \alpha+1)}
$$

and so called two parameter M-L function $\mathrm{E}_{\alpha, \beta}(\mathrm{z})$ :

$E_{\alpha, \beta}(z)=\sum_{k=0}^{\infty} \frac{z^{k}}{\Gamma(\alpha k+\beta)}$,

where $\alpha, \beta \in \mathbb{C}$ and $\operatorname{Re} \alpha>0, \operatorname{Re} \beta>0$. For $\alpha=\beta=1$ we obtain:

$$
E_{\alpha=1}(z)=E_{\alpha=1, \beta=1}(z)=\exp (z),
$$

(Haubold, Mathai \& Saxena, 2011).

The Laplace transforms of M-L functions are known, this fact facilitates solving some kinds of FDE on direct way using inverse Laplace transform (Podlubny, 1999).

To use state vector approach effectively (to omit integration in eq.10) the Bloch's equations $(8 \mathrm{a}, \mathrm{b}, \mathrm{c})$ have been converted to matrix form, and written in much comfortable homogeneous form (18):

$$
\begin{aligned}
& \frac{d}{d t}\left(\begin{array}{l}
M_{x} \\
M_{y} \\
M_{z} \\
M_{0}
\end{array}\right)=\left(\begin{array}{cccc}
-\frac{1}{T_{1}} & -\omega_{0} & \gamma B_{1} \sin \left(\omega_{1} t\right) & 0 \\
\omega_{0} & -\frac{1}{T_{2}} & -\gamma B_{1} \cos \left(\omega_{1} t\right) & 0 \\
-\gamma B_{1} \sin \left(\omega_{1} t\right) & \gamma B_{1} \cos \left(\omega_{1} t\right) & -\frac{1}{T_{2}} & \frac{1}{T_{1}} \\
0 & 0 & 0 & 0
\end{array}\right)\left(\begin{array}{l}
M_{x} \\
M_{y} \\
M_{z} \\
M_{0}
\end{array}\right)= \\
& =\boldsymbol{A}\left(\begin{array}{l}
M_{x} \\
M_{y} \\
M_{z} \\
M_{0}
\end{array}\right)
\end{aligned}
$$

or in matrix compact form (19):

$$
\frac{d \vec{M}(t)}{d t}=-\vec{A} \vec{M}(t) .
$$

If the matrix $\mathbb{A}$ is constant in time interval, adding the initial conditions $\mathbf{M}(\mathrm{t}=0)=\mathbf{M}(0)$ (magnetization at $\mathrm{t}=0$ time), we can obtain the solution of system (19) in an analytical form, in terms of the matrix exponential and initial states:

$\vec{M}(t)=e^{-\not{A} t} \vec{M}(0)$.

\section{Ordinary Order Bloch's Equations}

Just one particular case of the general system of Bloch's Equations (BE) (19) for both ordinary order (OOBE) and fractional order (FOBE) will be discussed in this paper.

Considered is the situation when no disturbance of $\mathrm{B}_{1}$ $\mathrm{RF}$ field occurs. It is a pure relaxation case, when position of the magnetization vector at the end of the resonance is the initial position of the magnetization vector after $\pi / 2$ pulse (Haacke et al., 1999). In this case the Bloch equation may be solved analytically for initial conditions $\operatorname{Mx}(0)=100, \operatorname{My}(0)=0, \operatorname{Mz}(0)=0$. The matrix A assumes the form:

$\boldsymbol{A}=\left(\begin{array}{cccc}-\frac{1}{T_{1}} & -\omega_{0} & 0 & 0 \\ \omega_{0} & -\frac{1}{T_{2}} & 0 & 0 \\ 0 & 0 & -\frac{1}{T_{2}} & \frac{1}{T_{1}} \\ 0 & 0 & 0 & 0\end{array}\right)$.

The solution of homogeneous linear system (19) reduces to the task of computing exponential matrix. To calculate the matrix exponential $\exp (\mathbb{A})$ the following classical method known from linear algebra (Lang, 2000) was used: after finding eigenvalues $\lambda_{\mathrm{i}}$ and corresponding eigenvectors a transformation to Jordan form was carried out. Because the exponential matrix for diagonal 
matrix is obtained by exponentiating each diagonal element we get the known solution for $\mathbf{M}(\mathrm{t})$ :

$$
\begin{aligned}
& \vec{M}(t)=\boldsymbol{Q} e^{t \boldsymbol{J}} \boldsymbol{Q}^{-1} \vec{M}(0)= \\
& =\left(\begin{array}{cccc}
e^{-\frac{1}{T_{2}} t} \cos \left(\omega_{0} t\right) & e^{-\frac{1}{T_{2}} t} \sin \left(\omega_{0} t\right) & 0 & 0 \\
-e^{-\frac{1}{T_{2}} t} \sin \left(\omega_{0} t\right) & e^{-\frac{1}{T_{2}} t} \cos \left(\omega_{0} t\right) & 0 & 0 \\
0 & 0 & e^{-\frac{1}{T_{1}} t} & 1-e^{-\frac{1}{T_{1}} t} \\
0 & 0 & 0 & 0
\end{array}\right)\left(\begin{array}{l}
M_{x} \\
M_{y} \\
M_{z} \\
M_{0}
\end{array}\right)
\end{aligned}
$$

where $\mathbb{Q}, \mathbb{Q}^{-1}$ are transformation matrices, $\mathbb{J}$ is a diagonal matrix.

All symbolic calculations were performed using the Matlab Symbolic Toolbox and MuPad computer algebra system (Symbolic Math Toolbox ${ }^{\mathrm{TM}} 5$, MuPAD ${ }^{\circledR}$ Tutorial, 1997-2008).

\section{Fractional Order Bloch's Equations}

The Bloch's equations (19) (OOBE) may now be converted formally to fractional form. The derivatives $\mathrm{d} / \mathrm{dt}$ in (19) are changed to fractional time derivative operators $\mathrm{d}^{\alpha} / \mathrm{dt}^{\alpha}$ in Caputo form ${ }^{C} \mathrm{D}_{\mathrm{t}}^{\alpha}$, where $0<\alpha<1$ is an arbitrary parameter representing the order of the fractional time derivative operator:

${ }_{a}^{C} D_{t}^{\alpha_{1}} M_{x}(t)=\omega_{0}^{\prime} M_{y}-\frac{M_{x}}{T_{2}^{\prime}}$

$C_{a} D_{t}^{\alpha}{ }^{2} M_{y}(t)=-\omega_{0}^{\prime} M_{x}-\frac{M_{y}}{T_{2}^{\prime}}$

${ }_{a}^{C} D_{t}^{\alpha_{3}} M_{x}(t)=-\frac{M_{0}-M_{z}(t)}{T_{1}^{\prime}}$

and where $\omega^{\prime}{ }_{0}$ and $T^{\prime}{ }_{1,2}$ are modified frequency and relaxation times, respectively. If we assume for simplicity, the values of $\alpha_{\mathrm{i}}$ are equal $\alpha_{\mathrm{i}}=\alpha$, then $\omega^{\prime}{ }_{0}$ and $\mathrm{T}{ }_{1,2}$ have the following form:

$$
\omega_{0}^{\prime}=\frac{\omega_{0}}{\tau_{2}^{\alpha-1}}, \frac{1}{T_{1}^{\prime}}=\frac{\tau_{1}^{1-\alpha}}{T_{1}}, \frac{1}{T_{2}^{\prime}}=\frac{\tau_{2}^{1-\alpha}}{T_{2}},
$$

The $\omega^{\prime}{ }_{0}$ and $\mathrm{T}_{1,2}$ are dependent on "fractionality" degree $\alpha$ and non-local fractional time $\tau$.

This form of FOBE (eqs. 23) was introduced in (Magin at al., 2009). It is worthy to mention that transformation from OOBE to FOBE is made on the pure heuristic (formal mathematical) base. Because the dimension of fractional derivative $\left[\mathrm{d}^{\alpha} / \mathrm{dt}^{\alpha}\right]=\mathrm{s}^{-\alpha}$, that means, $\mathrm{d}^{\alpha} / \mathrm{dt}^{\alpha}$ is not a pure time derivative operator in usual sense. In order to be consistent with time dimensionality (and magnetization dimension) a parameter $\tau$ is introduced in the following way:

$\left[\frac{1}{\tau^{1-\alpha}} \frac{d^{\alpha}}{d t^{\alpha}}\right]=s^{-1}$

and $\alpha$ has dimension of seconds $[\alpha]=\mathrm{s}$. Ordinary time derivative operator may therefore be changed to its fractional equivalent $\mathrm{d}^{\alpha} / \mathrm{dt}^{\alpha}$ having dimension $\mathrm{s}^{-1}$ and representing true time derivative operator. It was made by introducing a phenomenological parameter $\tau^{1-\alpha}$ (dimension $\mathrm{s}^{1-\alpha}$ ) representing non-local fractional time components of time transformation. Finally the transformation is given by the formula (Podlubny, 2002):

$\frac{d}{d t} \rightarrow \frac{1}{\tau^{1-\alpha}} \frac{d^{\alpha}}{d t^{\alpha}}$

If all $\alpha_{\mathrm{i}}$ have the same order $\alpha$, (commensurate order system), the previously introduced method of solving OOBE could be easily adapted to solve eqs. (23). After transformation of the system of equations $(23 a, b, c)$ to respective homogeneous form, the system matrix has the same form as in eq.(21). Only the values of parameters $\omega_{0}$ and $T_{1,2}$ are changed: $\omega_{0}->\omega_{0}$ and $T_{1,2^{-}}>T^{\prime}{ }_{1,2}$, respectively. Matrix $\mathbb{A}$ is diagonalizable, as was shown previously, and if we denote eigenvalues of $\mathbb{A}$ as $\lambda_{i}$, we get:

$$
\lambda_{1}=\left(\frac{-1}{T_{2}^{\prime}}-i \omega_{0}^{\prime}\right), \quad \lambda_{2}=\left(\frac{-1}{T_{2}^{\prime}}+i \omega_{0}^{\prime}\right), \quad \lambda_{3}=\left(\frac{-1}{T_{2}^{\prime}}-i \omega_{0}^{\prime}\right), \quad \lambda_{4}=\left(\frac{-1}{T_{1}^{\prime}}\right) .
$$

The Mittag-Leffler matrix function $\mathrm{E}\left(\mathbb{A}^{\alpha}\right)$ can be easy evaluated using following affinity transformation:

$E_{\alpha}\left(\boldsymbol{A} t^{\alpha}\right)=\sum_{k=0}^{\infty} \frac{\left(\boldsymbol{Q J}_{\alpha} \boldsymbol{Q}^{-1} t\right)^{k \alpha}}{\Gamma(\alpha k+1)}=\sum_{k=0}^{\infty} \frac{\left(\boldsymbol{Q}_{\alpha} \boldsymbol{Q}^{-1}\right)^{k \alpha} t^{k \alpha}}{\Gamma(\alpha k+1)}=\boldsymbol{Q} \boldsymbol{J}_{\alpha}^{M L} \boldsymbol{Q}^{-1}$

where $\mathbb{J}_{\alpha}$ is a Jordan form of $\mathbb{A}, \mathbf{J}^{\mathrm{ML}}{ }_{\alpha}$ is a diagonal matrix that contains the elements $\mathrm{E}_{\alpha}\left(\lambda_{\mathrm{i}} \mathrm{t}^{\alpha}\right)$ on its diagonal. The final form of matrix Mittag-Leffler function takes on the form: 
$E_{\alpha}\left(\not A t^{\alpha}\right)=\left(\begin{array}{cccc}\sigma_{2} & \frac{1}{2} i\left(\sigma_{2}-\sigma_{3}\right) & 0 & 0 \\ \frac{1}{2} i\left(-\sigma_{2}+\sigma_{3}\right) & \sigma_{1} & 0 & 0 \\ 0 & 0 & E_{\alpha}\left(\frac{-1}{T_{1}^{\prime}} t^{\alpha}\right) & 1-E_{\alpha}\left(\frac{-1}{T_{1}^{\prime}} t^{\alpha}\right) \\ 0 & 0 & 0 & 0\end{array}\right)$,

where

$\sigma_{1}=\frac{1}{2}\left(\sigma_{2}+\sigma_{3}\right), \quad \sigma_{2}=E_{\alpha}\left(\left(\frac{-1}{T_{2}^{\prime}}+i \omega_{0}^{\prime}\right) t^{\alpha}\right), \quad \sigma_{2}=E_{\alpha}\left(\left(\frac{-1}{T_{2}^{\prime}}-i \omega_{0}^{\prime}\right) t^{\alpha}\right)$

The general solution of the system $(23 a, b, c)$ can be now written as the linear combinations of Mittag-Leffler functions:

$$
\begin{aligned}
& M_{x}(t)=\frac{1}{2}\left[E_{\alpha}\left(\left(\frac{-1}{T_{2}^{\prime}}+i \omega_{0}^{\prime}\right) t^{\alpha}\right)+E_{\alpha}\left(\left(\frac{-1}{T_{2}^{\prime}}-i \omega_{0}^{\prime}\right) t^{\alpha}\right)\right] M_{x}(0)+ \\
& +\frac{1}{2} i\left[E_{\alpha}\left(\left(\frac{-1}{T_{2}^{\prime}}-i \omega_{0}^{\prime}\right) t^{\alpha}\right)-E_{\alpha}\left(\left(\frac{-1}{T_{2}^{\prime}}+i \omega_{0}^{\prime}\right) t^{\alpha}\right)\right] M_{y}(0)
\end{aligned}
$$

$M_{y}(t)=\frac{1}{2} i\left[E_{\alpha}\left(\left(\frac{-1}{T_{2}^{\prime}}-i \omega_{0}^{\prime}\right) t^{\alpha}\right)-E_{\alpha}\left(\left(\frac{-1}{T_{2}^{\prime}}+i \omega_{0}^{\prime}\right) t^{\alpha}\right)\right] M_{x}(0)+$ $+\frac{1}{2}\left[E_{\alpha}\left(\left(\frac{-1}{T_{2}^{\prime}}+i \omega_{0}^{\prime}\right) t^{\alpha}\right)+E_{\alpha}\left(\left(\frac{-1}{T_{2}^{\prime}}-i \omega_{0}^{\prime}\right) t^{\alpha}\right)\right] M_{y}(0)$,

$M_{z}(t)=E_{\alpha}\left(\frac{-1}{T_{1}^{\prime \prime}} t^{\alpha}\right) M_{z}(0)+\left[1-E_{\alpha}\left(\frac{-1}{T_{1}^{\prime}} t^{\alpha}\right)\right] M_{0}$.

When we put $\alpha=1$ into eqs. (30), and use eq.(17) we get the solution of ordinary order Bloch's equations in known form:

$$
\begin{aligned}
& M_{x}(t)=e^{\frac{-1}{T_{2}}}\left(M_{x}(0) \cos \left(\omega_{0} t\right)-M_{y}(0) \sin \left(\omega_{0} t\right)\right), \\
& M_{y}(t)=e^{\frac{-1}{T_{2}}}\left(M_{y}(0) \cos \left(\omega_{0} t\right)-M_{x}(0) \sin \left(\omega_{0} t\right)\right), \\
& M_{z}(t)=M_{z}(0) e^{\frac{-1}{T_{1}}}+\left(1-e^{\frac{-1}{T_{1}}}\right) M_{0},
\end{aligned}
$$

which corresponds with the solution given by eq. (22).

\section{ADOMIAN DECOMPOSITION METHOD (ADM)}

Confirmation of the previously obtained solution can be made using Adomian Decomposition Method (ADM). This analytical (semi-analytical) method introduced by Adomian in eighties of XX century is generally used to solve ordinary and partial differential equations, both linear and nonlinear (Adomian,1994), also to solve FDE (Duan, Rachb, Baleanu \& Wazwaz, 2012). In ADM the general nonlinear equation is written in decomposed form:

$\widehat{L} u(t)+\widehat{R} u(t)+\widehat{N} u(t)=g$,

where $\mathrm{L}^{\wedge}$ is invertible linear operator (usually highest order derivative in equation), $\mathrm{R}^{\wedge}$ is remaining linear part, $\mathrm{N}^{\wedge}$ represents a nonlinear operator and $\mathrm{g}$ a wellbehaved function. The solution is splitted on the linear part and non-linear part. The general solution is searched in a form of an infinite series which converges to accurate solutions. The nonlinear contribution is obtained in a form of so-called "Adomian polynomials" from its expansion into the power series. When applying inverse operator $\mathrm{L}^{-1}$ to eq.(32) we get:

$u(t)=f(t)-\widehat{L}^{-1} \widehat{R} u(t)-\widehat{L}^{-1} \widehat{N} u(t)$,

where

$u(t)=f(t)-\widehat{L}^{-1} \widehat{R} u(t)-\widehat{L}^{-1} \widehat{N} u(t)$.

The general solution $\mathrm{u}(\mathrm{t})$ is expressed in series form:

$u=\sum_{k=0}^{\infty} u_{k}$

and the action of nonlinear part $\mathrm{N}^{\wedge}$ is given as a series of Adomian polynomials $\mathrm{A}_{\mathrm{n}}$, which are functions of consecutive approximations $\mathrm{u}_{1}, \mathrm{u}_{2}, \ldots, \mathrm{u}_{\mathrm{n}}$ :

$\widehat{N} u=\sum_{n=0}^{\infty} A_{n}$.

Adomian polynomials can be calculated from relation:

$$
A_{n}=\frac{1}{n !} \frac{d^{n}}{d \lambda^{n}}\left[\widehat{N} \sum_{k=0}^{\infty} \lambda^{k} u^{k}\right]_{\lambda=0}
$$

Finally, we obtain solution $\mathrm{u}(\mathrm{t})$ of eq.(32) as in a form of recurrent relation, successive terms of Adomian series have form: 
$u_{0}=f(t), u_{k+1}=-\widehat{L}^{-1} \widehat{R} u_{k}-\widehat{L}^{-1} A_{n}$.

ADM technique provides an analytical solution without discretization or linearization, and its true power is seen in solving various nonlinear problems. The Adomian method has many modifications oriented to specific applications, some of them are widely used to solve the FDE, both ordinary and partial (Duan et al., 2012; Junsheng, Jianye \& Mingyu, 2007). Modified $\mathrm{ADM}$ is easy to formulate in the context of our problem. Let consider system (23), here written in matrix form as:

${ }_{\mathbb{a}} D_{t}^{\mathbb{A}} \vec{M}(t)=-A \vec{M}(t)$

with previously introduced initial condition $\mathbf{M}(\mathrm{t}=0)=\mathbf{M}(0)$. System (39) is linear and homogeneous, therefore the non-homogeneity and nonlinear part in ADM approach cannot be taken under consideration. Integrating eq.(39) according the definition of Caputo derivative ${ }^{C} \mathrm{D}^{\alpha}$ (5) we get:

$$
I^{\alpha C}{ }_{a} D_{t}^{\alpha} \vec{M}(t)=\int_{0}^{\tau} d \tau_{2} \frac{\left(t-\tau_{2}\right)^{\alpha-1}}{\Gamma(\alpha)} \int_{0}^{\tau_{2}} \frac{\left(t-\tau_{1}\right)^{\alpha-1}}{\Gamma(1-\alpha)} \vec{M}\left(\tau_{1}\right) d \tau_{1}
$$

Integrals in eq.(40) could be evaluated after changing their order and using known integral relation (Whittaker \& Watson, 1965):

$$
\int_{\tau}^{t}\left(t-\tau_{1}\right)^{a-1}\left(\tau_{1}-\tau\right)^{b-1} d \tau_{1}=\frac{\Gamma(a) \Gamma(b)}{\Gamma(a+b)}(t-\tau)^{a+b-1} .
$$

After rearrangement the final result yields:

$I^{\alpha C} D_{t}^{\alpha} \vec{M}(t)=\vec{M}(t)-\vec{M}(0)$.

In spirit of $\mathrm{ADM}$ method we decompose $\mathbf{M}(\mathrm{t})$ into the infinite series:

$\vec{M}(t)=\sum_{k=0}^{\infty} \vec{M}_{A(k)}$,

where $\mathrm{M}_{\mathrm{A}(\mathrm{k})}$ is a $\mathrm{k}$-th element of series (35). The first element of the expansion (43) denoted here as $\mathbf{M}_{\mathrm{A}(0)}$ is given by initial condition:

$\vec{M}_{A(0)}(t)=\vec{M}(0)$

As we see the partial solution is given by iteration procedure:
$\vec{M}_{A(k)}(t)=I^{\alpha} \not \mathcal{A} \vec{M}_{A(k-1)}(t), k>1$.

In the next step we obtain k-th part of Adomian series as:

$$
\vec{M}_{A(k)}(t)=A^{k} I^{\alpha} \vec{M}_{A(0)}(t) .
$$

Summation the series gives the magnetization in terms of Mittag-Lefller function:

$\vec{M}(t)=\sum_{k=0}^{\infty} \frac{\boldsymbol{A}^{k} t^{k \alpha}}{\Gamma(k \alpha+1)} \vec{M}(0)=E_{\alpha}\left(\not \boldsymbol{A} t^{\alpha}\right) \vec{M}(0)$.

The result (47) corresponds with previously achieved solution, when state vector method was used (eqs. 30). The ADM is a general and powerful method and could also serve to verify results obtained for Bloch's equations in a non-homogeneous form, as they are usually presented. The ADM method has an additional advantage, using ADM method, it is easy to perform the calculation with the Riemann-Liouville derivatives. When we use the RL derivatives ${ }^{\mathrm{RL}} \mathrm{D}^{\alpha}$ instead of Caputo's in eq.(39), we must change the initial conditions, the new initial condition has following form:

$\left(I^{1-\alpha R L} D_{t}^{\alpha} \vec{M}(t)\right)_{t=0}=\vec{M}(0)$.

Using integral operator $\mathrm{I}^{\alpha}$ to both sides of eq.(39), definition of ${ }^{\mathrm{RL}} \mathrm{D}^{\alpha}$ derivative (4) and initial condition (47) we get:

$I^{\alpha R L} D_{t}^{\alpha} \vec{M}(t)=\vec{M}(t)-\frac{t^{1-\alpha}}{\Gamma(\alpha)} \vec{M}(0)=I^{\alpha} \not A \vec{M}(t)$

and the solution takes the form:

$\vec{M}(t)=\frac{t^{1-\alpha}}{\Gamma(\alpha)} \vec{M}(0)+I^{\alpha} \not \vec{A}(t)$.

Using Adomian method with zero approximation:

$\vec{M}_{A(0)}(t)=\frac{t^{1-\alpha}}{\Gamma(\alpha)} \vec{M}(0)$,

we obtain as previously the series:

$\vec{M}(t)=\sum_{k=0}^{\infty} \boldsymbol{A}^{k} t^{k \alpha} \frac{t^{1-\alpha}}{\Gamma(\alpha)} \vec{M}(0)=\sum_{k=0}^{\infty} \boldsymbol{A}^{k} \frac{t^{k \alpha+\alpha-1}}{\Gamma(\alpha)} \vec{M}(0)$,

which factorizes to final solution: 


$$
\begin{aligned}
& \vec{M}(t)=t^{1-\alpha} E_{\alpha, \alpha}\left(\not A t^{\alpha}\right) \vec{M}(0)=E_{\alpha, 1}\left(\not A t^{\alpha}\right) \vec{M}(0)= \\
& =E_{\alpha}\left(\not A t^{\alpha}\right) \vec{M}(0) .
\end{aligned}
$$

The result agrees with these given by eq.(47) and eq.(30).

\section{ASYMPTOTIC SOLUTION OF FRACTIONAL ORDER BLOCH'S EQUATIONS}

The general solutions of FOBE (30) are given in the form of complex valued functions. The complex valued M-L functions are generally difficult in examination (Haubold at al.,2001; Hilfer \& Seybold, 2006). In order to investigate the fractional character of relaxation described by eqs. (30) the asymptotic behavior of M-L functions was examined. For $\arg |z| \leq \pi / 2$, with accuracy to $\mathrm{z}^{-\mathrm{k}}$ the following asymptotic formula can be deduced from integral representation of M-L function (Gorenflo, Loutchko \& Luchko, 2002):

$$
E_{\alpha}(z)=\frac{1}{\alpha} \exp \left(z^{\frac{1}{\alpha}}\right)-\sum_{k=1}^{p} \frac{z^{-k}}{\Gamma(1-\alpha k)}+O\left(|z|^{-1-p}\right) .
$$

Let introduce notations:

$$
\lambda=\frac{-1}{T_{2}^{\prime}}+i \omega_{0}^{\prime}=r e^{i \varphi}
$$

where $r=|\lambda|$ and $\varphi=\arg (\lambda)$ :

$$
r=\sqrt{\left(\frac{1}{T_{2}^{\prime}}\right)^{2}+\left(\omega_{0}^{\prime}\right)^{2}}, \quad \varphi=\tan ^{-1}\left(\omega_{0}^{\prime} T_{2}^{\prime}\right)
$$

Considering only the first term in eq.(54), that is the situation corresponding to long time approximation $\left(\omega_{\mathrm{o}}{ }^{\prime} \mathrm{T}_{2}{ }^{\prime}>1\right.$, in NMR), we obtain asymptotic solutions of equations $(30 \mathrm{a}, \mathrm{b}, \mathrm{c})$ for magnetization components in the form of complex valued functions of time. After some transformations they can be converted to factorized exponential form easier to analyze and plot:

$$
\begin{aligned}
& M_{x}(t)=\frac{1}{\alpha} \exp \left[-r^{\frac{1}{\alpha}} \cos \left(\frac{\varphi}{\alpha}\right) t\right] \cos \left[r^{\frac{1}{\alpha}} \sin \left(\frac{\varphi}{\alpha}\right) t\right] M_{x}(0)+ \\
& +\frac{1}{\alpha} \exp \left[-r^{\frac{1}{\alpha}} \cos \left(\frac{\varphi}{\alpha}\right) t\right] \sin \left[r^{\frac{1}{\alpha}} \sin \left(\frac{\varphi}{\alpha}\right) t\right] M_{y}(0)
\end{aligned}
$$

$$
\begin{aligned}
& M_{y}(t)=\frac{1}{\alpha} \exp \left[-r^{\frac{1}{\alpha}} \cos \left(\frac{\varphi}{\alpha}\right) t\right] \cos \left[r^{\frac{1}{\alpha}} \sin \left(\frac{\varphi}{\alpha}\right) t\right] M_{y}(0)- \\
& -\exp \left[-r^{\frac{1}{\alpha}} \cos \left(\frac{\varphi}{\alpha}\right) t\right] \sin \left[r^{\frac{1}{\alpha}} \sin \left(\frac{\varphi}{\alpha}\right) t\right] M_{y}(0) .
\end{aligned}
$$

In a case of $\mathrm{Mz}(\mathrm{t})$ component the asymptotic expansion valid for small $\mathrm{z}$, is a "stretched exponential":

$$
E_{\alpha}\left(-z^{\alpha}\right) \square \exp \left(-\frac{z^{\alpha}}{\Gamma(1+\alpha)}\right)
$$

Using this expansion we get asymptotic expansion for $\mathrm{Mz}(\mathrm{t})$ :

$$
M_{z}(t)=\exp \left(\frac{-t^{\alpha}}{T_{1}^{\prime} \Gamma(1+\alpha)}\right) M_{z}(0)+\left[1-\exp \left(\frac{-t^{\alpha}}{T_{1}^{\prime} \Gamma(1+\alpha)}\right)\right] M_{0}
$$

For $\alpha=1$, the solution $(57 \mathrm{a}, \mathrm{b})$ has the same form as in previously obtained eqs.(31), the result is easy to check using known formula:

$$
\cos \left(\tan ^{-1}(t)\right)=\frac{1}{\sqrt{1+t^{2}}}, \quad \sin \left(\tan ^{-1}(t)\right)=\frac{t}{\sqrt{1+t^{2}}},
$$

and properties of the Gamma function. The calculated magnetization components (eqs.57) are given as the real functions of time and the results show, that in the long time approximation the system (30) demonstrates the fractional order dynamics: precession with attenuation and phase shift, fractional order relaxation. Both components of solution, exponential function (damping) and the oscillation part are modulated by functions depending on order of differentiation $\alpha$ representing the "fractionality" of the system. Influence of "fractionality" on time behavior of magnetization components is shown in Fig.1 and Fig.2. 

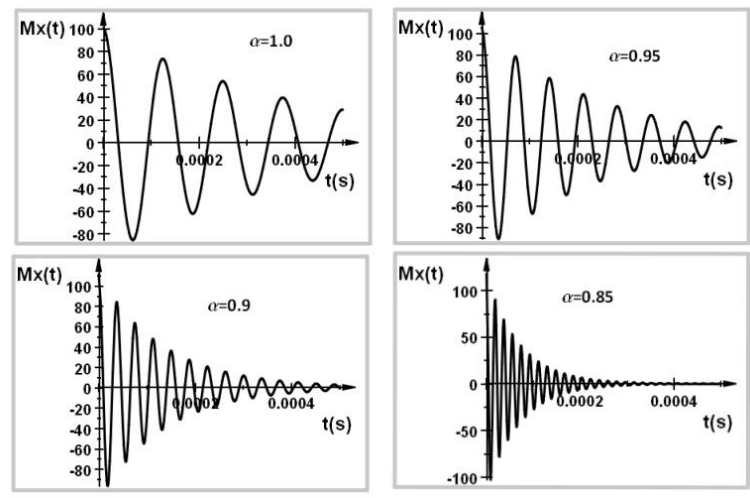

Fig.1. Influence of fractional order $\alpha$ on time behavior of $\mathrm{Mx}$ component of magnetization $\mathbf{M}$. $\mathrm{Mx}=\mathrm{Mx}(0)=100, \mathrm{~T}_{2}{ }^{\prime}=$ $20(\mathrm{~ms})^{\alpha}, \mathrm{f}_{0}=160 \mathrm{~Hz}$. For these the eqs. $(57 \mathrm{a}, \mathrm{b})$ were used.

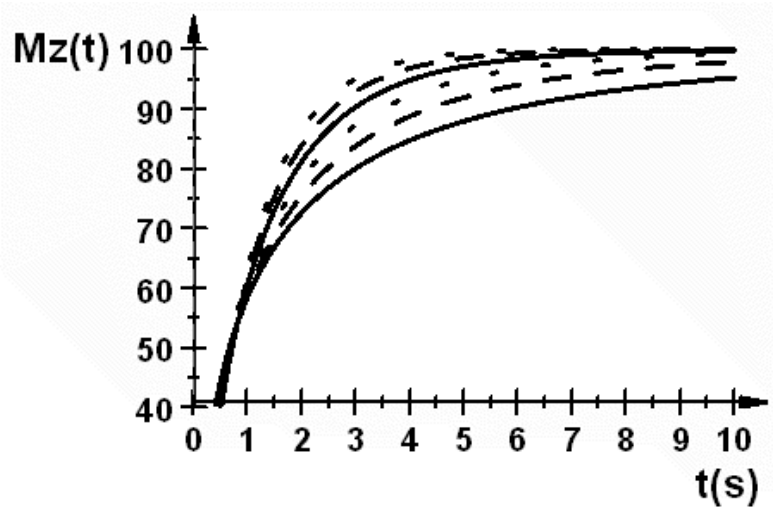

Fig.2. Influence of fractional order $\alpha$ on time behavior of $\mathrm{Mz}$ component of magnetization $\mathbf{M} . \mathrm{Mz}=\mathrm{Mz}(0)=0, \mathbf{M}_{0}=100, \mathrm{~T}_{1}{ }^{\prime}=$ $1(\mathrm{~s})^{\alpha},, \mathrm{f}_{0}=160 \mathrm{~Hz} . \alpha$ is in the range from $\alpha=0.5$ (bottom curve) to $\alpha=1$ (upper curve). For these the eq. (57c) was used.

\section{DIRECT INTEGRATION OF FRACTIONAL ORDER BLOCH'S EQUATIONS}

\section{Numerical Evaluation of Mittag-Leffler Functions}

A numerical solution of FOBE includes numerical evaluation the values of Mittag-Leffler functions and numerical approximations of fractional derivatives.

The time behavior of magnetization components during relaxation (30) is described by Mittag-Leffler functions. For M-L functions $\mathrm{E}_{\alpha}(\mathrm{z})$ and $\mathrm{E}_{\alpha, \beta}(\mathrm{z})$ evaluation of their values of for complex arguments $\mathrm{z} \epsilon$ $\mathbb{C}$ is a very complicated and unexplored problem (Hilfer at al., 2006). To calculate numerically the M-L functions values from their series representation a direct method of truncation can be used, in most cases with success, but when the series slowly converges or does not converges, some more sophisticated algorithms must be employ. Some algorithms were elaborated to solve this problem (Gorenflo at al, 2002; Diethelm, Ford, Freed \& Luchko, 2005). One of most popular implementation of such algorithms, applied also in this work to check the validity of the asymptotic solution of FOBE (data not shown), is Matlab file mlf.m (Podlubny, Kacenak, 2005).

\section{Dynamical System Approach}

Often, a simpler way to get the solution of differential equations is to choose the direct integration of the state equations. Special methods developed in theory of dynamical systems and optimal control theory, e.g. in construction of PID (Proportional-Integral-Derivative) regulators are very useful from the practical point of view. The fractional derivatives in this case are evaluated in the frequency domain (as filters) opposite to usual time domains. (Duarte \& da Costa, 2013). Several groups have improved this method, most known implementations are Matlab Toolboxes CRONE (Oustaloup, Mathieu \& Lanusse, 1995) and NINTEGER (Duarte, 2007).These Toolboxes have their own GUI (Graphical User Interface) and may be linked to Matlab and Simulink, a special graphical environment oriented at simulation of dynamical systems (Simulink, 19902013). In Simulink, the differential equations and initial conditions are represented by special blocks and flow diagrams. Dynamical problems are solved by direct integration of the state equations using various kinds of numerical algorithms (Gran, 2007). The results are easy to plot, including phase diagrams. This approach is universally accepted in optimal control theory to simulate regulations systems, also of fractional nature (Monje at al., 2010, Mital, Kr, \& Prasad 2011, Zhao, Zhao \& Luo, 2011). First, to simulate Bloch's equations this method was introduced in (Petráš, 2010; Petráš, 2011), where the Simulink schemas realizing integration of OOBE and FOBE were presented.

The integrated equations have the form of eqs. $(23, \mathrm{a}, \mathrm{b}, \mathrm{c})$ and if $\alpha_{1}=\alpha_{2}=\alpha_{3}=1$ we deal with OOBE case, when orders in derivatives are different the schema describes the general fractional system FOBE.

Integration of FOBE (eqs.23) is performed using standard algorithms which are connected to specific blocks integr_Mi(t), where $\mathrm{i}=\mathrm{x}, \mathrm{y}, \mathrm{z}$. Fractional derivatives are realized using block nid (Duarte, 2005). To solve the state equations for FOBE having the form of eqs. (60), the integration was performed using standard Runge-Kutta method of 4-th order implemented in Simulink. The integration procedure is realized within block Integer_Mi(t) (the other integration methods are also available in Matlab and could be used instead Runge- Kutta method, if necessary). Simulink schemes to perform integration of FOBE is presented in Fig.3.

$$
M_{x}(t)={ }_{0}^{C} D_{t}^{1-\alpha_{1}}\left(\int_{0}^{t}\left[\omega_{0}^{1} M_{y}(t)-\frac{M_{x}(t)}{T_{2}^{\prime}}\right] d t\right),
$$




$$
\begin{aligned}
& M_{y}(t)={ }_{0}^{C} D_{t}^{1-\alpha_{2}}\left(\int_{0}^{t}\left[-\omega_{0}^{1} M_{x}(t)-\frac{M_{y}(t)}{T_{2}^{\prime}}\right] d t\right), \\
& M_{x}(t)={ }_{0}^{C} D_{t}^{1-\alpha_{3}}\left(\int_{0}^{t}\left[\frac{M_{0}-M_{z}(t)}{T_{1}^{\prime}}\right] d t\right)
\end{aligned}
$$

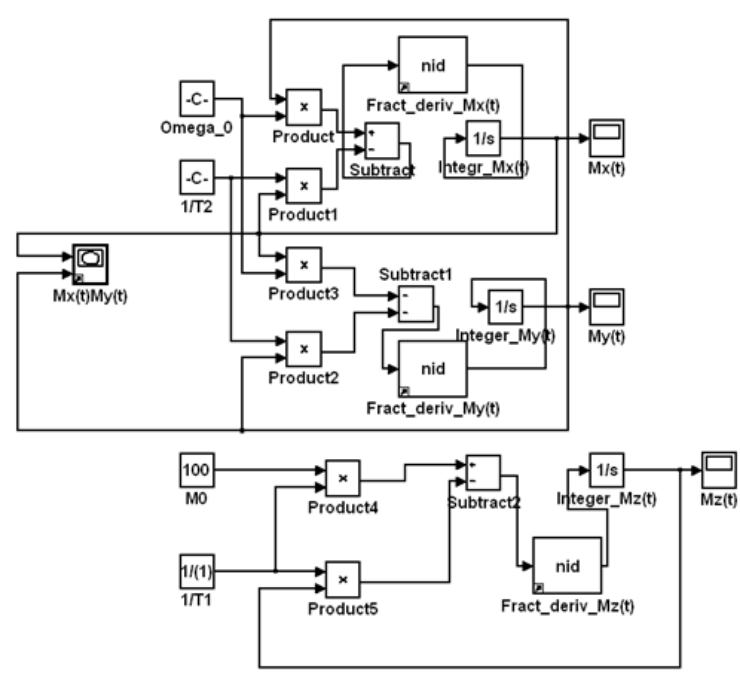

Fig.3.Simulink realization of FOBE integration (according to Petráš, 2010)

The other option to obtain solution of FOBE numerically is the direct integration of Bloch's equations using the definition of fractional derivatives in Grünwald-Letnikov ${ }^{\mathrm{GL}} \mathrm{D}^{\alpha}$ form. The Matlab code FOBlochEqs based on the following integration schema (61) was also published (Petráš, 2011):

$M_{x}\left(t_{k}\right)=\left(\omega_{0}^{\prime} M_{y}\left(t_{k}-1\right)-\frac{M_{X}\left(t_{k}-1\right)}{T_{2}^{\prime}}\right) h^{\alpha_{1}}-\sum_{j=0}^{k} c_{j}^{\left(\alpha_{1}\right)} M_{X}\left(t_{k-1}\right)$,

$M_{y}\left(t_{k}\right)=\left(-\omega_{0}^{\prime} M_{x}\left(t_{k}-1\right)-\frac{M_{y}\left(t_{k}-1\right)}{T_{2}^{\prime}}\right) h^{\alpha_{2}}-\sum_{j=0}^{k} c_{j}^{\left(\alpha_{2}\right)} M_{y}\left(t_{k-1}\right)$,

$M_{z}\left(t_{k}\right)=\left(\frac{M_{0}-M_{Z}\left(t_{k}-1\right)}{T_{1}^{\prime}}\right) h^{\alpha_{3}}-\sum_{j=0}^{k} c_{j}^{\left(\alpha_{3}\right)} M_{x}\left(t_{k-1}\right)$,

where $\mathrm{h}$ denotes an integration step, $\mathrm{c}_{\mathrm{j}}^{\left({ }^{(\alpha)}\right)}$ are combinatorial factors (Petráš, 2011). These codes were used to check the results obtained by the analytical methods and Simulink simulations.

It is easy to see that this numerical scheme enables us to solve the FOBE for different values of $\alpha_{\mathrm{i}}$. Examples of simulations results showing the dependence of magnetization as a function of fractional derivative order are presented in Fig.4 and Fig.5. The figures present the phase plots 3D and 2D, for different values of differentiation order $\alpha$. That permits to imagine the connection between "fractionality" and relaxation.
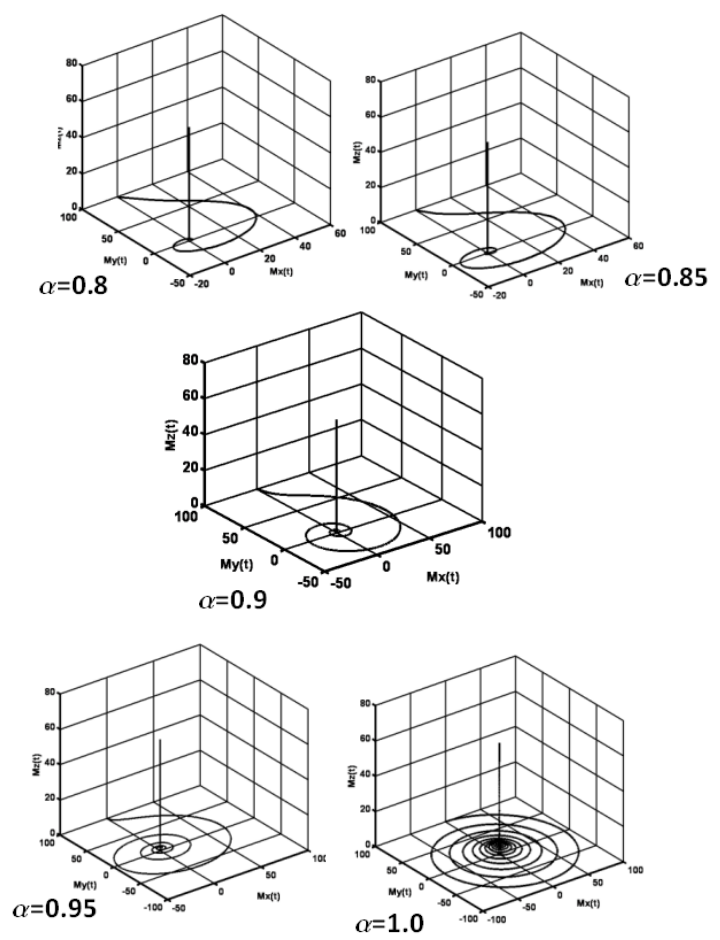

Fig.4. Numerical realization of FOBE integration (eqs.60) in 3D space $(M x(t)$ vs. $M y(t)$ vs. $M z(t)$ as function of order of fractional derivative $\alpha$. Simulation time $1 \mathrm{~s} . \mathrm{Mx}=\mathrm{Mx}(0)=100$, $\mathrm{T}_{2}{ }^{\prime}=20(\mathrm{~ms})^{\alpha}, \mathrm{f}_{0}=160 \mathrm{~Hz}$. 

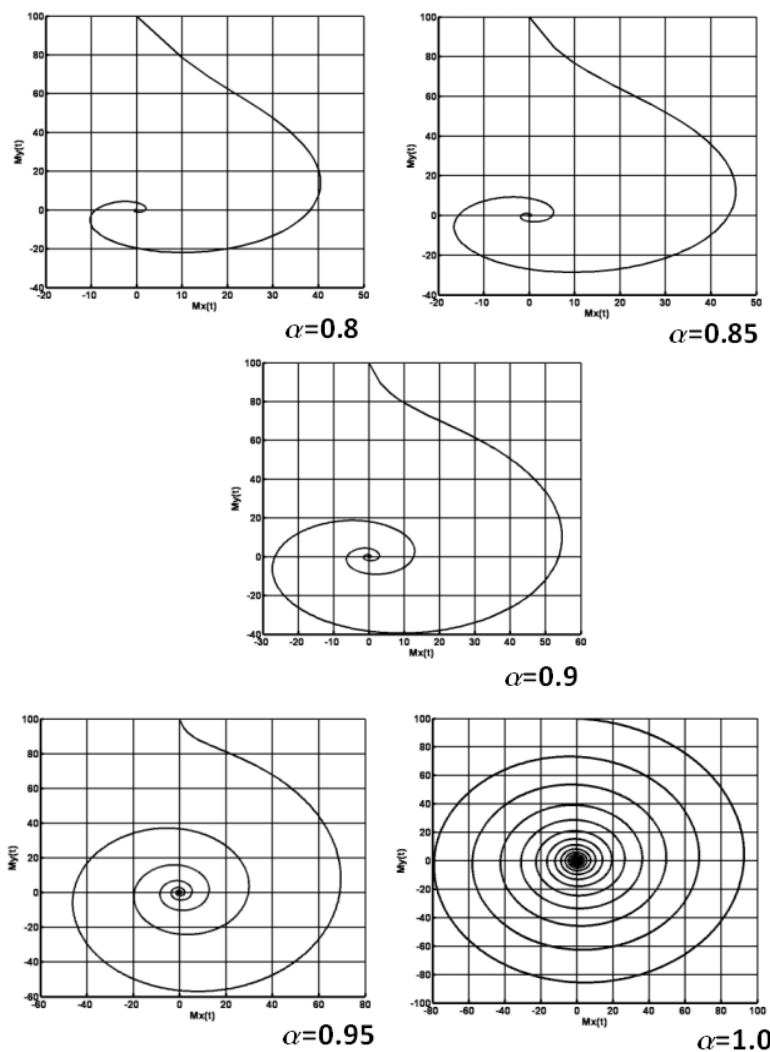

Fig.5. Numerical realization of FOBE integration (eqs.60) in 2D space $\mathrm{My}(\mathrm{t})$ vs. $\mathrm{Mx}(\mathrm{t})$ as function of order of fractional derivative $\alpha$. Simulation time $1 \mathrm{~s} . \mathrm{Mx}=\mathrm{Mx}(0)=100, \mathrm{~T}_{2}{ }^{\prime}=$ $20(\mathrm{~ms})^{\alpha}, \mathrm{f}_{0}=160 \mathrm{~Hz}$.

\section{DISSCUSSION}

At the work an attempt to solve Fractional Order Bloch's Equations in the systematic way is presented. The generalization of Ordinary Order Bloch's Equations to fractional order equations was first made in (Magin $e t$ al.,2009 ) using Laplace transform method; solutions of FOBE were obtained in terms of Mittag-Leffler functions.

In this work, a new approach is presented. The FOBE system was transformed into matrix form, to the homogeneous system of equations. This simplified application of the general methods of optimal systems theory and obtaining analytical solutions. The solutions were given in a compact form as a linear combination of $\mathrm{M}-\mathrm{L}$ functions. The answers received have other formal form than those obtained at earlier recalled work (Magin et. al., 2009), but it is easy to convert one form of the solutions into the other. For $\alpha=1$, when the M-L functions convert oneself to ordinary exponents, both forms of the solutions are transformed to form typical for the solution of the OOBE $(31 a, b, c)$.

As the next method for obtaining a solution of FOBE in the analytical form, an Adomian Decomposition Method in matrix form was suggested. This method also led to solution given in analytical form, agreeing with results received previously, with help of state function method. Obtaining of the analytical solutions of FOBE is made much easier, when we apply both of the methods to BE equations given in the homogeneous form, since we avoid an additional troublesome integration (we are not obliged to calculate convolution integrals).

The obtained form of expression for magnetization components $(57 \mathrm{a}, \mathrm{b}, \mathrm{c})$ has also an additional benefit. It has the relatively simple form of the asymptotic expansion and expressions for magnetization components have the factorized form of exponentials and trigonometric functions. These expressions have a general character similar to the solutions of the corresponding OOBE (eq.22) and comparing the forms of both solutions it is easy to see the modulatory influence of the fractional derivative order $\alpha$ on the individual elements of function, which describe magnetization as a function of time. Both relaxation (exponential) part and oscillating part of FOBE solution include new internal exponential and oscillating element (scaling parts). That means that usual time constant is replaced by time function and process tends to move forward in time as coefficient $\alpha$ in exponent change from $\alpha=1$ to $\alpha=0.8$ (Fig.1,2,4,5). These observations are consistent with the interpretations of the fractional derivatives in chronological time as averages over the fluctuating operational time, and therefore phase space trajectories are not energy level curves but rather spirals into the origin, demonstrating dissipative character of internal processes (Stanislavsky, 2004).

In the work, the emphasis was put on the analytical methods. Numerical methods, developed by other authors give results easy to present graphically, but all numerical procedures take advantage of so-called "short memory principle" (Podlubny,1999). On the strength of the "short memory-principle", time behavior of $f(t)$ in a recent past only is taken into account. This fact necessarily changes the actual problem. The principle was introduced in order to analyze FDE using previously elaborated methods, but it consequently changes the solution, which may be physically incorrect. It is obvious, that we need an analytical method which would offer a control of the procedure of FDE solution and would guarantee the physically correct solution. It seems that ADM method fulfils these requirements, because it treats the response of the physical system on external stimuli in a systematic way. The decomposed parts of ADM are related physically to system reactions, and what is important in solving FDE, the ADM involves the initial conditions as the initial approximation. Just the simplest case of using ADM to solving of FOBE was demonstrated here. 
It is an advantage of the ADM, that it may be applied to both kinds of fractional derivatives, as it was shown (eqs. 47,53). Having many different approaches to the same problem enhances the chances to elucidate the fundamental unresolved problem of fractional calculus applications - unambiguous physical interpretations of the fractional derivatives (Podlubny, 2002; Stanislavsky, 2004). It is known that the order of the fractional derivative $0<\alpha<1$ classifies and quantifies the influence of previous history of process. For unity value of the fractional order (or integer order of one) the influence of history is minimal, in the sense that, then the evolution enters only through the present state. Small value of $\alpha$ corresponds to a strong influence of history. These facts can be easy drawn from the analysis of kernel $\Phi_{\alpha}(\mathrm{t})$ in eq. (5):

$$
I^{\alpha} f(t)=\Phi_{\alpha} * f(t)=\frac{t^{\alpha-1}}{\Gamma(\alpha)} * f(t), \quad t>0, \alpha>0
$$

where * denotes convolution. When $\alpha \rightarrow 0$ then $\Phi_{\alpha}(\mathrm{t}) \rightarrow$ $\delta(\mathrm{t})$ (Dirac's function) and system loses its memory, in opposite case when $\alpha \rightarrow 1$ then $\Phi_{\alpha}(\mathrm{t}) \rightarrow \mathrm{H}(\mathrm{t})$ (step Heaviside's function), the system has full memory.

\section{CONCLUSIONS}

Bloch's equations are an excellent model for testing the influence of history on dynamics. Nevertheless, further development of fractional magnetic relaxation theory at molecular level would probably demand formulating the problem of magnetic relaxation in terms of fractional quantum mechanics. However, it seems that it is much more important to find suitable experimental systems in mesoscopic scale in which the effects of memory and disorder would be manifest. Magnetic spectroscopies, both NMR and EPR, with well elaborated theories and opportunities for measurement within many orders of magnitudes in a time scale are ideal candidates to give a vital contribution in the rapidly growing area of fractional physics.

\section{REFERENCES}

Adomian G.(1994). Solving Frontier Problems of Physics: The Decomposition Method. Kluwer Academic Publishers, Dordrecht/Boston/London.

Alexander S., Entin - Wohlman O. \& Orbach R. (1985). Relaxation and nonradiative decay in disordered systems. I. One-fracton emission. Phys. Rev. B., 32(10), 6447-6455.

Alexander S., Entin - Wohlman O. \& Orbach R. (1986). Relaxation and nonradiative decay in disordered systems. II. Two-fracton inelastic scattering. Phys. Rev. B., 33(6), 39353946.
Butzer P.L. \& Westphal U. (2000). An Introduction to Fractional Calculus. [In:] Hilfer. R. (ed).,Applications of Fractional Calculus in Physics., World Scientific Publishing Co. Pte. Ltd., Singapore.,1-85.

Diethelm K., Ford N.J., A.D. Freed A.D., Luchko Yu. (2005). Algorithms for the fractional calculus: A selection of numerical methods, Comput. Methods Appl. Mech. Engrg. 194, 743-773.

Diethelm K. (2010). The Analysis of Fractional Differential Equations. An Application-Oriented Exposition Using Differential Operators of Caputo Type. Springer, Heidelberg Dordrecht London New York.

Duan J-S., Rachb R., Baleanu D., Wazwaz A-M. (2012). A review of the Adomian decomposition method and its applications to fractional differential equations., Communications in Fractional Calculus,3(2), 73-99.

Duarte V. (2005) http://web.ist.utl.pt/ duarte.valerio/ ninteger/ninteger.htm.

Duarte V. \& Sa da Costa J.(2013). An Introduction to Fractional Control. The Institution of Engineering and Technology. London, United Kingdom.

Feldman Y., Puzenko A. \& Ryabov Y. (2006). Dielectric Relaxation Phenomena [In:] Coffey W.T \& Kalmykov Y.P. (eds.) Complex Materials Fractals, Diffusion, and Relaxation in Disordered Complex Systems. A Special Volume of Adv. in Chem. Phys. 133(A)., John Wiley \& Sons, Inc., Hoboken, New Jersey,pp. 1-125.

Gorenflo R., Loutchko I., Luchko Yu., (2002). Computation of the Mittag-Leffler function $\mathrm{E}_{\mathrm{a}, \mathrm{b}}(\mathrm{z})$ and its derivatives, Fract. Calculus Appl. Anal. 5, 491-518, erratum, (2003) 6, 111112.

Gran R. J. (2007). Numerical Computing with Simulink. Vol.I. Creating Simulations. SIAM. Society for Industrial and Applied Mathematics, Philadelphia, USA.

Haacke E.A., Brown R.B., Thompson M.R., Venkatesan R. (1999). Magnetic Resonance Imaging. Physical Principles and Sequence Design. J. Wiley \& Sons, Inc., New York Chichester Weinheim Brisbane Singapore Toronto.

Haubold H.J, Mathai A.M. \& Saxena R.K. (2011). MittagLeffler Functions and Their Applications. Review Article. $J$. Applied Mathematics Vol.2011, Article ID 298628, 51 pages. (http://dx.doi.org/10.1155/2011/298628).

Herrmann R. (2011). Fractional Calculus. An Introduction for Physicists. World Scientific Publishing Co. Pte. Ltd., Singapore.

Hilfer R. Fractional Time Evolution, (2000). An Introduction to Fractional Calculus. [In:] Hilfer. R. (ed.). Applications of Fractional Calculus in Physics., World Scientific Publishing Co. Pte. Ltd., Singapore.87- 130.

Hilfer R \& Seybold H.J. (2006). Computation of the generalized Mittag-Leffler function and its inverse in the complex plane, Integral Transforms and Special Functions. 17(9), 637-652.

Junsheng D., Jianye A., Mingyu X. (2007). Solution of System of Fractional Differential Equations by Adomian Decomposition Method, Appl. Math. J. Chinese Univ. Ser. B., 22(1),7-12.

Kaczorek T.(2011). Selected Problems of Fractional Systems Theory. Springer-Verlag Berlin Heidelberg.

Kilbas A.A., Srivastava H.M., Trujillo J.J. (2006). Theory and Applications of Fractional Differential Equations., Elsevier B.V., Amsterdam, The Netherlands. 
Lang S. (2000). Linear Algebra.3-ed., Springer-Verlag, New York Inc.

Lewis F.L., Syrmos V.L. (1995).Optimal Control.2-nd ed., John Wiley \& Sons, Inc., New York.

Madhu P.K., Kumar A. (1995). Direct Cartesian-space solutions of generalized Bloch equations in the rotating frame. J. Magn. Reson. A, 114, 201-211.

Magin R., Feng X., Baleanu D., (2009) Solving the fractional order Bloch equation, Concepts in Magnetic Resonance Part A., 34A (1) 16-23.

Metzler R., Klafter R. (2000).The Random Walk's Guide to Anomalous Diffusion: A Fractional Dynamics Approach. Physics Reports. 339, 1-77.

Miller K.S, Ross B. (1993). An Introduction to the Fractional Calculus and Fractional Differential Equations. John Wiley \& Sons, Inc., New York.

Mital P.B, Kr U. \& Prasad R.C. (2011). Modeling and Simulation of Fractional Order Chaotic Systems Using Matlab/Simulink. VSRD-IJEECE, 1(4), 220-224.

Monje C.A, Chen YQ., Vinagre B.M, Xue D., Feliu V. (2010). Fractional-order Systems and Controls. Fundamentals and Applications. Springer-Verlag London Limited.

Oldham K.B., Spanier J. (1974). The Fractional Calculus. Theory and Applications of Differentiation and Integration to Arbitrary Order. Academic Press, Inc., San Diego, California.

Oustaloup A., Mathieu B., and Lanusse P. (1995). The CRONE control of resonant plants: application to a flexible transmission. Eur. J. of Control, 1(2), 113-121. (http://www.ims-bordeaux.fr/CRONE/toolbox).

Podlubny, I. (1999). Fractional differential equations. Academic Press, San Diego.

Podlubny I. (2002).Geometrical and physical interpretation of fractional integration and fractional differentiation. Fractional Calculus \& Applied Analysis. 5(4),367-386.

Podlubny I, Kacenak M. (2005). Mittag-Leffler function, [PodlubnyI.,online],http://www.mathworks.com/matlabcentr al/fileexchange/8738.

Petráš I. (2010). Modeling and numerical analysis of fractional-order Bloch equations, Computers and Mathematics with Application., 61, 341-356.
Petráš I. (2011). Fractional-Order Nonlinear Systems. Modeling, Analysis and Simulation. Higher Education Press, Beijing and Springer-Verlag Berlin Heidelberg.

Sabatier J., Agrawal O.P., Tenreiro-Machado J.A.(eds.) (2007). Advances in Fractional Calculus.Theoretical Developments and Applications in Physics and Engineering. Springer, Dordrecht, The Netherlands.

Simulink ${ }^{\circledR} \quad$ Getting Started Guide (1990-2013).The MathWorks, Inc.

Slichter C.P. (1989). Principles of Magnetic Resonance. Third Enlarged and Updated Edition. Springer-Verlag Berlin Heidelberg New York London Paris Tokyo Hong Kong.

Stanislavsky A.A. (2004). Probability Interpretation of the Integral of Fractional Order. Theoretical and Mathematical Physics., 138(3),418-431.

Symbolic Math Toolbox ${ }^{\mathrm{TM}} 5$, MuPAD ${ }^{\circledR}$ Tutorial.(1997-2008). The MathWorks, Inc.

Torrey H.C. (1949). Transient nutations in nuclear magnetic resonance, Phys. Rev. 76, 1059-1068.

Uchaikin V.V. (2013). Fractional Derivatives for Physicists and Engineers. Vol. I. Background and Theory. SpringerVerlag, Heidelberg Dordrecht London New York.

West B.J. (2006). Fractal Physiology, Complexity, and the Fractional Calculus. [In:] Coffey W.T \& Kalmykov Y.P. (eds.) Complex Materials Fractals, Diffusion, and Relaxation in Disordered Complex Systems. A Special Volume of Adv. in Chem. Phys. 133(B), 1-92., John Wiley \& Sons, Inc., Hoboken, New Jersey.

Weilbeer M., "Efficient Numerical Methods for Fractional Differential Equations and their Analytical Background". PhD. Thesis, Carl-Friedrich-Gauß-Fakultät für Mathematik und Informatik der Technischen Universität Braunschweig. 2005.

Whittaker E.T, and G.N. Watson G.N (1965). A Course of Modern Analysis., Cambridge University Press, Cambridge, (polish. ed. PWN, Warszawa, 1967).

Yariv A.(1975). Quantum Electronics. John Wiley \& Sons, New York.

Zhao C., Zhao Y. Luo L. (2011). Fractional Modeling Method Research on Education Evaluation, Journal of Software, 6(5),901-907. 